\title{
La decisión de jubilar. Dificultades y factores asociados en personal universitario de Concepción ${ }^{1}$
}

\author{
Nieves Stuardo ${ }^{2}$, Mercedes Zavala ${ }^{3}$, José Manuel Merino ${ }^{4}$
}

\begin{abstract}
A consecuencia del cambio demográfico, en la mayoría de los países del mundo, la expectativa de vida se ha incrementado. Junto con ello, se prevé un aumento del número de años de vida económicamente activa. En esto inciden también, los deficientes niveles de tasa de reemplazo en las pensiones que hace que las personas mayores posterguen su retiro laboral. Este artículo muestra los resultados de una investigación cuyo propósito fue conocer y analizar la dificultad para la decisión de jubilar y los factores asociados a ello. No obstante, se trabajó con una aproximación cuantitativa que correlaciona variables, se formula una pregunta abierta. Los resultados obtenidos mostraron que las variables consideradas no influyen en forma estadísticamente significativa en la dificultad para la decisión de jubilar; a pesar de ello, el análisis cualitativo permitió establecer nuevas categorías de variables, entre ellas los incentivos económicos asociados a la jubilación, que, por su frecuencia, determinaron en su momento la decisión de jubilar.
\end{abstract}

Palabras clave: decisión de jubilar, conflicto decisional, jubilación, dificultad.

\section{A Decisão de aposentar-se. Dificuldades e fatores associados em pessoal universitário de Concepción}

\section{RESUMO}

Como consequência das alterações demográficas, na maioria dos países do mundo a expectativa de vida tem aumentado. Junto a isso, se prevê um aumento do número de anos de vida economicamente ativa. Nisto também incidem os deficientes níveis de taxas de substituição nas pensões, que fazem que os idosos adiem sua retirada do mundo laboral. Este artigo mostra os resultados duma investigação cujo propósito foi conhecer e analisar

1 Artículo recibido: 07/06/2016. Artículo aprobado: 28/09/2016.

2 Chilena. Asistente Social. Universidad de Concepción, Chile. Correo electrónico: nieves.stuardo@gmail.com

3 Chilena. Enfermera. Universidad de Concepción, Chile. Correo electrónico: mzavala@udec.cl

4 Chileno. Sociólogo. Universidad de Concepción, Chile. Correo electrónico: jmerino@udec.cl 
a dificuldade da decisão de aposentar-se e os fatores associados à mesma. Contudo, trabalhou-se com uma aproximação quantitativa que correlaciona variáveis, se formula uma pergunta aberta. Os resultados obtidos mostram que as variáveis consideradas não influem de maneira significativa estatisticamente na dificuldade da decisão de aposentar-se; contudo, a análise qualitativa permitiu estabelecer novas categorias de variáveis, entre elas, os incentivos econômicos associados com a aposentadoria, que pela sua frequência determinaram, na ocasião, a decisão de aposentar-se.

Palavras-chave: decisão de aposentar-se, conflito decisional, aposentadoria, dificuldade

\section{The decision of retirement. Difficulties and associated factors in a university's staff in Concepción}

\section{ABSTRACT}

As a result of demographic change, life expectancy has increased in most countries of the world. Along with this, an increase in the number of years of economically active life is expected. This also affects the poor levels of pension replacement rate that causes older people to postpone their retirement. This article shows the results of an investigation which purpose was to understand and analyze the difficulty of the decision to retire and the factors associated with it. However, we worked with a quantitative approximation that correlates variables, formulating an open-ended question. The obtained results showed that the variables considered do not influence in a statistically significant way on the difficulty of the decision to retire; despite this, qualitative analysis allowed us to establish new categories of variables, including the economic incentives associated with retirement, which, by their frequency, determined at the time the decision to retire.

Key words: decision to retire, decisional conflict, retirement, difficulty

\section{Antecedentes}

La escalada del envejecimiento es enorme en todo el mundo. Las posibilidades de alargar la vida parecen ilimitadas. Los procesos de envejecimiento son muy distintos en cada país, debido a la heterogeneidad en el avance de la transición demográfica; el prematuro proceso en los países industrializados se tradujo en un envejecimiento más avanzado. De acuerdo a informaciones de la Oficina de Estadísticas de las Comunidades Europeas (EUROSTAT) cerca del $15 \%$ de la población de los 25 países de la Comunidad Europea, tenía 65 años y más a principios del siglo XXI. Europa ha 
aumentado progresivamente el índice de esperanza de vida al nacer que va desde los 80 a 81,5 para los países con mayor población envejecida (CEPAL, 2011).

En las últimas décadas los países de América Latina han registrado profundas transformaciones demográficas, como la disminución de la tasa de crecimiento y el progresivo envejecimiento de la población. Esta evolución responde a la acelerada baja de la fecundidad y al descenso sostenido de la mortalidad.

A mediados del siglo pasado, la tasa de crecimiento anual de la población regional era de 2,8\%, mientras que en la actualidad es de sólo 1,2\%. De acuerdo con las proyecciones de la CEPAL, serán 656 millones en 2025 y 707 millones en 2040.

Para 2025 la CEPAL proyecta que las personas mayores de 60 años serán poco más de 98 millones. En 2050 uno de cada cuatro latinoamericanos será mayor de 60 años. Aunque hay diferencias por países. Bolivia, Guatemala, Haití, Honduras, Nicaragua y Paraguay muestran un envejecimiento incipiente. En Brasil, Colombia, Costa Rica, Ecuador, El Salvador, México, Panamá, Perú y Venezuela se vive un envejecimiento moderado, mientras que Argentina y Chile están un paso más allá. Cuba y Uruguay presentan ya un envejecimiento avanzado (Bárcena, 2011).

Este contexto plantea significativos retos para el conjunto de la sociedad. Se requiere ampliar la protección de las personas que hoy son mayores. Frente a esta situación, muchos países están reaccionando favorablemente, aplicando pensiones sociales como Panamá, Argentina, Chile y El Salvador, que generan un ingreso mínimo pero estable en la vejez, programas que son una herramienta muy útil en la lucha contra la pobreza y en la revaloración de la contribución de las personas mayores dentro de cada país. Estos cambios reflejan un aumento de la cobertura de los sistemas de pensión no contributivos (HelpAge International, 2014).

Chile vive también un incremento sostenido de la población total y particularmente del tramo sobre 60 años, si se considera que en 1950 éste representaba sólo un 6,9\%; esta cifra alcanza hoy un 16,7 
de adultos mayores que viven en Chile (Casen, 2013). Si se lleva esto al ámbito productivo, la tendencia en la actividad laboral de las personas mayores ha concentrado la atención durante los últimos años en las regiones desarrolladas. En varios países de la Organización de Cooperación y Desarrollo Económico (OCDE) se puede observar un estancamiento de la tendencia al retiro temprano de la fuerza laboral.

Esto quizás, como uno de los impactos positivos que han ocurrido con el cambio demográfico, no solo en Chile sino también en otras partes del mundo, dado que las personas están llegando a la edad legal de jubilar, en condiciones de salud y energía como para continuar aportando con su trabajo, y adquiriendo nuevos conocimientos que les permiten reinventarse. A esto contribuye también, el envejecimiento activo, promovido por la OMS, lo que ha abierto el debate para ver los desafíos que las políticas públicas deberán considerar como una buena oportunidad para las personas y la población que envejece (OMS, 1999). El envejecimiento activo se aplica tanto a los individuos como a los grupos de población, y se entiende como el proceso de optimización de las oportunidades de salud, participación y seguridad con el fin de mejorar la calidad de vida a medida que las personas envejecen (OMS, 1999).

En consecuencia, uno de los desafíos que plantea el envejecimiento de la población y que requiere de una atención particular es el de garantizar pensiones suficientes, financieramente viables $y$ adaptables a las condiciones cambiantes de la economía a todas las personas que han cumplido su vida laboral y se encuentran en edad de retiro, a fin de evitar la amenaza de la pobreza para las personas mayores (OISS, 2011).

Ante esta situación global, en el futuro, los gobiernos que manejan la edad de jubilación a los 65 años, ofrecerán pensiones que mantendrán a las personas en nivel de pobreza. Tener un estilo de vida superior supondrá una responsabilidad personal (Leeson, 2013).

Sin embargo, esta problemática está presente, dado que muchas personas no desean jubilarse a la edad legal por el bajo monto de 
sus pensiones. Un ejemplo de esta situación son los servicios públicos, que han debido implementar incentivos al retiro, como ha sido el caso de los sectores de educación y salud, entre otros (Lorenzini, 2014).

En el centro de la protección social chilena se encuentra el sistema de pensiones y jubilaciones. Chile fue el primer país de América Latina que creó un Sistema de Seguridad Social, a comienzos del siglo XX. A lo largo de los años se fueron creando diversos regímenes de pensiones, diferenciados por el tipo de actividad o grupos ocupacionales, con reglas y beneficios distintos. Es así como llegan a coexistir 52 "Cajas" o Instituciones de Previsión, las que operaban bajo el esquema de reparto. Esto significa que los aportes de los afiliados activos financiaban las pensiones de los pasivos y, por tanto, la subsistencia del sistema estaba supeditada a la relación "trabajador/pensionado" existente en la población en cada momento del tiempo (Asociación de AFP Chile).

Durante los primeros años de existencia del sistema, la proporción de trabajadores fue suficiente para financiar los beneficios de los pensionados. Sin embargo, los cambios demográficos -que fueron reflejando una permanente disminución de la natalidad y un aumento en las expectativas de vida- revirtieron esta relación, provocando un fuerte desfinanciamiento del sistema.

Mientras que en el año 1955 por cada 12,2 trabajadores cotizantes había 1 pensionado, en 1980 por cada 2,5 trabajadores cotizantes había 1 pensionado. Es decir, sólo en 25 años el costo para los trabajadores cotizantes se incrementó casi 5 veces.

El desfinanciamiento y la inequidad del esquema de reparto dieron origen a una reforma previsional que creó, mediante el D.L. 3.500 de 1980, un nuevo sistema de pensiones basado en la capitalización individual y administrado por entidades privadas denominadas Administradoras de Fondos de Pensiones (AFP). El Antiguo Sistema continuó funcionando, principalmente a través de un ente único denominado Instituto de Normalización Previ- 
sional (INP), hoy Instituto de Previsión Social (IPS), el cual fusionó a las principales Cajas de Previsión (Asociación de AFP Chile).

El Estado se hizo responsable del financiamiento de las cotizaciones pagadas en el Antiguo Sistema por aquellas personas que se cambiaron al Nuevo Sistema, a través del instrumento Bono de Reconocimiento, que se reajusta de acuerdo a la variación de la inflación y devenga un interés del $4 \%$ real anual, que se capitaliza cada año (Asociación de AFP Chile).

Varios países, especialmente de América Latina y Europa del Este, han adoptado reformas similares. Aun cuando este tipo de sistemas tiene una serie de fortalezas comparado con los tradicionales esquemas de reparto, por sí solos no resuelven el financiamiento de la vejez o invalidez en poblaciones donde hay una mala distribución del ingreso. En el caso de Chile, el diagnóstico que se hizo el año 2006 mostró que existía una parte importante de la población que no tendría ahorros suficientes para el financiamiento de su vejez. Esto se debía principalmente a la baja densidad de cotizaciones que se explica, en el caso de los hombres, por el trabajo independiente y la informalidad en el mercado laboral. En el caso de las mujeres, la participación en el mercado laboral en Chile es especialmente baja, por lo que existían en promedio largos períodos en que ellas no registraban cotizaciones (Supen, 2010).

En marzo de 2008 fue promulgada y publicada en el Diario Oficial la ley de la Reforma Previsional, uno de los cambios más profundos que ha tenido el sistema de pensiones chileno en muchos años, reforzado por el esquema de Tres Pilares. El primero, conocido como Pilar Solidario, apunta a proteger a aquellas personas que no ahorraron para su vejez en ningún sistema de pensiones o lo hicieron en magnitud insuficiente, financiado con recursos públicos; el segundo, conocido como Pilar Contributivo, de ahorro obligatorio y capitalización individual; y el Pilar Voluntario, donde los cotizantes pueden realizar ahorro de forma planificada y voluntaria, de acuerdo a las necesidades de pensión o de anticipación de la jubilación que tengan. 
La ley estableció una secuencia en el tiempo para la vigencia plena de la normativa, partiendo con las disposiciones contempladas para el Pilar Solidario -que comenzó a regir en julio de 2008-, con el pago de la Pensión Básica Solidaria para todas aquellas personas que, por algún motivo, no cotizaron en ningún sistema de pensiones. En 2012, y en forma gradual, comenzaron a incorporarse los trabajadores independientes al sistema de pensiones, integrándose al Pilar Contributivo o de ahorro obligatorio y capitalización (Asociación de AFP Chile).

Adicionalmente, los beneficios del Pilar Solidario se integran con los autofinanciados a partir de la cuenta individual de pensiones, estableciendo un Aporte Previsional Solidario (APS) que se otorga en forma decreciente en relación con el monto ahorrado, bajo el supuesto de que esto mejoraría los incentivos a contribuir. El actual Sistema Integrado de Pensiones, por lo tanto, incluye beneficios autofinanciados y subsidiados, por vejez, invalidez y sobrevivencia, que permiten abordar los objetivos de suavizar el consumo y aliviar la pobreza en la vejez (Informe Comisión Presidencial Pensiones, 2015).

No existe contribución del empleador al fondo de pensiones, salvo para la realización de trabajos calificados como pesados y en el caso del Seguro de Invalidez y Sobrevivencia (SIS), que es financiado por los empleadores durante la vida laboral activa de los afiliados. El sistema mantiene en los beneficios autofinanciados las modalidades de pensión establecidas en el D.L. 3.500. El sistema continúa sin incorporar a las Fuerzas Armadas, de Orden y Seguridad y Carabineros, cuyo régimen se financia de imposiciones y recursos provenientes del aporte fiscal (Informe Comisión Presidencial Pensiones, 2015).

En cuanto a los resultados del sistema, estudios de la Organización de Cooperación y Desarrollo Económico (OCDE) indican que las jubilaciones en Chile corresponden a un 51,8\% del último sueldo, mientras que el promedio de los países de la OCDE es $64,1 \%$, es decir, bajo el promedio; en tanto, si se compara con 
los países pertenecientes a la agrupación que lideran en tasas de reemplazo, Holanda y Hungría, que ofrecen a sus jubilados el equivalente al $101 \%$ y $95 \%$ de sus últimos sueldos, respectivamente.

Otro de los datos recalcados por la OCDE en materia de pensiones, fue que la edad de retiro en Chile en las mujeres alcanza los 70,4 años, cuando lo establecido legalmente es 60, siendo la mayor diferencia entre la edad oficial y efectiva de jubilación entre los 34 países de la OCDE. Las personas en Chile están retrasando su edad de jubilación, debido a que la tasa efectiva de reemplazo es muy baja.

Otra consecuencia de este fenómeno es que la edad establecida para pensionarse es a los 65 años, lo que se toma como referencia para que a una persona se la defina como de "edad avanzada", lo que sucede en muchos países europeos al igual que en Chile. Sin embargo, "esta edad está siendo disociada de la edad biológica" (García, 2010).

Esta discrepancia entre la edad biológica y la edad laboral hace que haya grandes diferencias individuales en la adaptación a la jubilación, en el sentido que para algunos puede significar simplemente el final de la vida laboral, un nuevo papel en la sociedad y el comienzo de la vejez; para otros una liberación del estrés laboral; sin embargo, para otros, puede significar una pérdida personal, ya que al sentirse todavía productivos, desean seguir siendo activos (García, 2010), situación que en Chile se discute cada vez con mayor frecuencia, y que va a la par con un cuestionamiento al sistema de pensiones.

Otro de los aspectos importantes en el sistema de pensiones tiene que ver con la información y conocimiento del sistema por parte de los afiliados, en tópicos como: descuento para las pensiones, los beneficios a los que tienen derecho por invalidez o sobrevivencia del afiliado; el conocimiento de las edades legales de retiro para pensión y el de parámetros importantes como los montos y requisitos de las pensiones mínimas y asistenciales, lo que quedó 
en evidencia con la $1^{\text {a }}$ Encuesta de Protección Social realizada en el 2002 (EPS, 2002).

Frente a este escenario, ¿cuáles son las principales dificultades que enfrentan las personas para tomar la decisión de jubilar, y qué otros factores individuales han sido determinantes en una decisión de carácter único e irrepetible?

En su dimensión más básica, un proceso de toma de decisión puede entenderse como la elección de lo "mejor" entre lo "posible". Ahora bien, según se defina "qué es lo mejor y qué es lo posible, la persona se enfrentará a distintas situaciones de decisión" (Begoña, 2007).

La jubilación se define como "la fase final del ciclo vital de vida ocupacional de una persona, así como el periodo siguiente después de haber culminado una carrera laboral, y se presenta de manera simultánea con otros cambios en la vida de una persona" (Villa, 2005). Desde este punto de vista la jubilación es un proceso y su duración está determinada por diversos sucesos que comienzan antes y pueden concluir años después (Rincón, 2011).

Desde esta mirada la decisión de jubilar también puede tener impacto según el momento en que se concrete, así lo demuestra el estudio de panel "Efectos causales del tiempo de jubilación sobre la salud mental y física de las personas", de Esteban Calvo, realizada en Estados Unidos en el 2012, cuyos resultados mostraron que las jubilaciones tempranas, que ocurren antes de la edad legal, disminuyen la salud.

Otro hallazgo importante plantea que los trabajadores que gozan de buena salud tienden a jubilarse más tarde, y que los que están más satisfechos con su trabajo y se inquietan por cambios en la legislación de la Seguridad Social tienden a retirarse antes, según estudio "Factores que influyen en la decisión de jubilar del personal de la Universidad Autónoma de San Luis Potosí, Colombia”, en el marco de cambios en el sistema de pensiones de 27 universidades (Izar et al., 2010). 
La jubilación puede representar una crisis personal, a veces intensa en su etapa inicial, debido a que se produce a una edad en la que la mayoría de las personas está bien física e intelectualmente y bastantes desearían mantener su vida profesional por más tiempo (Bruguera, 2011).

La ocurrencia de pérdidas y ganancias caracteriza todo el ciclo vital, y se pone de manifiesto tanto en las etapas que tradicionalmente han sido consideradas sólo de pérdidas (la vejez) como en aquellas que únicamente han sido concebidas en función de las ganancias (la infancia) (Villar, 2001). Desde esta teoría la jubilación para algunas personas puede ser vivida con satisfacción personal y para otras como una verdadera pérdida.

Basándose en la bibliografía examinada, el propósito general de esta investigación fue analizar la dificultad para la decisión de jubilar y los factores que están asociados a ésta, en personas que se acogieron a retiro de las Universidades de Concepción y Universidad Católica de la Santísima Concepción. Se prevé que muchas personas no jubilan a la edad legal por el bajo monto de sus pensiones; si bien este factor es el más conocido, no se han encontrado estudios en relación a otros factores que determinan que las personas se acojan a jubilación o permanezcan trabajando por más tiempo. En esta investigación no se incluyó una mirada desde la familia, sin embargo, en los hallazgos los encuestados señalaron que jubilarían para estar con la familia.

Un primer objetivo apuntó a conocer las características sociodemográficas de las personas jubiladas, y su correlación con la dificultad para la decisión de jubilar; un segundo objetivo buscó identificar el nivel de conocimiento previsional que tenían las personas sobre el sistema de pensiones antes de su jubilación; y por último, relacionar el nivel de satisfacción que muestran las personas frente a su sistema de pensiones, con la dificultad en la decisión de jubilar. 


\section{Metodología}

Se realizó un estudio cuantitativo, descriptivo y correlacional, con muestreo de tipo no probabilístico, considerando a personas jubiladas de 2 instituciones educacionales de la VIII Región. La unidad de análisis correspondió a hombre o mujer jubilados, que en su vida laboral activa se desempeñaban en la Universidad de Concepción o en la Universidad Católica de la Santísima Concepción, y que se acogieron a jubilación entre los años 2011 al 2014. La muestra quedó constituida por 34 jubilados, que voluntariamente accedieron a participar en la Encuesta, de un total de 179. La información se obtuvo utilizando la técnica Survey Social, con apoyo de muestreo de bola de nieve, que se usa para acceder a personas de difícil acceso por parte del investigador, en la que los individuos seleccionados para ser estudiados reclutan a nuevos participantes entre sus conocidos (Ochoa, 2015).

La variable dependiente corresponde a la dificultad para la decisión de jubilar, entendida la decisión como un "acto deliberado, consciente y racional tendiente a lograr un estado futuro" (Simon, 1957). El evento de la jubilación puede representar una crisis para la persona, pues afecta al individuo en su contexto global, en lo biológico, psicológico, social y espiritual, por tanto, se genera un conflicto decisional, el que se define como un estado de incertidumbre acerca de cuál curso de acción tomar debido a que las diferentes alternativas ofrecen beneficios y riesgos (Urrutia et al., 2008).

Las variables predictoras corresponden a: los aspectos demográficos, el nivel de conocimiento previsional que las personas tenían antes de jubilar, y el grado de satisfacción de los encuestados con el sistema previsional al que pertenecen.

Para la recolección de datos se utilizó un instrumento que consta de 4 partes, una primera con 18 preguntas, dirigidas a obtener información sociodemográfica, edad, sexo, estado civil, nivel educacional, categoría ocupacional, lugar de trabajo, 
número de años trabajados, previsión actual, estado de salud; presencia de enfermedades, actividades recreativas, participación en grupos.

La segunda parte consistió en un cuestionario con 8 preguntas orientadas a conocer el nivel de conocimiento previsional al momento de jubilar, para lo cual se categorizaron las respuestas con valores de a) $=1, \mathrm{~b}$ ) $=2$ y c) $=3$ puntos, desde el menor al mayor conocimiento previsional de las personas. Las categorías de respuesta para estas preguntas corresponden a la siguiente escala de puntuación: $1=$ menor conocimiento previsional; $2=$ mediano conocimiento previsional; y 3 = mayor conocimiento previsional. Además, esta segunda parte contiene 2 preguntas orientadas a conocer el nivel de satisfacción que tienen las personas respecto de su sistema previsional y el monto de las pensiones que perciben, estableciendo categorías de respuestas que tienen un valor de $a)=3, b)=2$ y c) $=1$ punto, desde la mayor a menor satisfacción con el sistema, de acuerdo a la siguiente escala: $1=$ mayor satisfacción; $2=$ medianamente satisfecho y $3=$ menor satisfacción.

Para medir la variable dependiente "dificultad para la decisión de jubilar" se utilizó una escala de Conflicto decisional "Mi dificultad en tomar esta decisión". La escala de conflicto decisional (ECD) fue adaptada de la Decisional Confíct Scale (DCS), instrumento que se basa en el Modelo conceptual de apoyo a la toma de decisiones en salud de Ottawa y su propósito es identificar los conflictos decisionales y los factores que los influyen. Fue validada en 1995 por su autora Annette O’Connor, de la Escuela de Enfermería, Facultad de Ciencias de la Salud, Departamento de Epidemiología y Salud Comunitaria, Facultad de Medicina, Universidad de Ottawa, Canadá.

En Chile esta escala es validada en la versión en español por Mila Urrutia, Solange Campos, y su autora Annette O'Connor, de la Universidad Católica de Chile, entre los años 1999 y 2004. La escala está conformada por 16 ítems para respuestas en formato 
Likert de 5 puntos (desde 1, total acuerdo, a 5, total desacuerdo), en que los puntajes altos indican conflicto decisional y factores contribuyentes. Para medir la dificultad en la decisión de jubilar se utilizaron las primeras 12 preguntas de la escala, con un puntaje mínimo de 12 y un máximo de 60 puntos. El puntaje obtenido en cada caso permitió conocer el nivel de dificultad que los encuestados presentaron en el momento de tomar la decisión de jubilar.

La cuarta parte del instrumento implicó una pregunta abierta: ¿cuáles fueron los factores más importantes considerados por Ud. para tomar la decisión de jubilar?

Las respuestas y relatos de los encuestados fueron grabados y transcritos en forma separada. Se procedió al análisis cualitativo de los datos obtenidos durante la observación y la entrevista. Se realizó en primer lugar, la lectura de cada una de las transcripciones y en cada una se seleccionaron fragmentos relacionados, que para fines de ordenamiento se agruparon por temas y por entrevistados. Dichos temas se establecieron de acuerdo al objetivo de la pregunta. Enseguida se dio lectura a cada fragmento, y se procedió a la selección de segmentos que se constituyeron en las unidades de análisis, las que se codificaron con un número correlativo (desde I hasta XVIII).

Se procedió a analizar las variables predictoras utilizadas para conocer en qué medida cada una de ellas ha tenido relación en la decisión de jubilar, o simplemente la decisión no está influida por alguna de estas variables, sino que por otras no consideradas en este estudio.

Los datos fueron recolectados entre los meses de octubre 2015 a enero de 2016, y se analizaron con el paquete IBM SPSS Statistics versión 19. 
La decisión de jubilar. Dificultades y factores asociados en personal universitario de Concepción / Stuardo

\section{Resultados}

Las características de la muestra estudiada se presentan en la siguiente tabla:

Tabla No 1 . Características de la Muestra

\begin{tabular}{|l|l|l|l|}
\hline & & $\mathrm{N}$ & $\%$ \\
\hline $\begin{array}{l}\text { Institución a la que } \\
\text { pertenece }\end{array}$ & Universidad de Concepción & 20 & 58,82 \\
\hline & Universidad Católica Ssma. Concepción & 14 & 41,17 \\
\hline Sexo & Hombres & 21 & 61,76 \\
\hline & Mujeres & 13 & 38,23 \\
\hline Estado Civil & Solteros & 4 & 11,42 \\
\hline & Casados & 24 & 70,58 \\
\hline & Viudos & 2 & 5,88 \\
\hline $\begin{array}{l}\text { Nivel } \\
\text { Educacional }\end{array}$ & Divorciados & 4 & 11,42 \\
\hline & Educac. Universitaria & 26 & 76,47 \\
\hline & Educac. Secundaria o técnica completa & 05 & 14,70 \\
\hline Categoría & Educac. Secundaria o técnica incompleta & 03 & 8,82 \\
\hline Ocupacional & Profesionales & 22 & 64,70 \\
\hline & Profesionales con postgrado & 04 & 11,76 \\
\hline & Administrativos y técnicos & 04 & 11,76 \\
\hline & Personal de servicio & 04 & 11,76 \\
\hline Año de Jubilación & 2011 & 10 & 29,41 \\
\hline & 2012 & 11 & 32,35 \\
\hline & 2013 & 10 & 29,41 \\
\hline & 2014 & 3 & 8,82 \\
\hline Religión & Católica & 25 & 73,52 \\
\hline & Otras & 9 & 26,47 \\
\hline & & & \\
\hline
\end{tabular}

Fuente: Tesis de Magíster "La decisión de jubilar, dificultades y factores asociados en Personal Universitario de Concepción”. Marzo 2016.

La percepción que los encuestados tienen de su estado de salud es buena (50\%) y muy buena (32\%), sólo un 20,6\% presenta discapacidad física limitante. 
Fue importante en este estudio el nivel educacional de los encuestados, dado que se observó una concentración en personas con educación universitaria $(76,47 \%)$, de éstos un $73,0 \%$ se desempeñó en el cargo de académico. Los resultados del nivel educacional y de la categoría ocupacional permiten ver una característica de homogeneidad, dado el tamaño de muestra pequeña.

Al realizar el análisis de datos y modelización estadística bivariada se encontró que la variable dependiente "dificultad para la decisión de jubilar" presenta un valor modal de 24 , y una media de 24,15 , en una puntuación de la escala de 12 a 60. Esto adquiere relevancia puesto que, bajo la media, se concentró el $67 \%$ de las personas, y sólo el $32 \%$ se ubicó sobre la media, lo que indica que las personas presentaron un nivel menor de dificultad para tomar la decisión de jubilar.

La decisión de jubilar se considera una etapa compleja y sus dificultades pueden estar asociadas a múltiples factores que pueden ser de tipo sociodemográfico, al mayor o menor conocimiento previsional que poseen las personas, y también al grado de satisfacción que tienen con su sistema previsional. Dado que la variable dependiente "dificultad para la decisión de jubilar" no tiene una distribución normal, se utilizaron pruebas estadísticas no paramétricas. En el caso de diferencias entre dos medias, se utilizó la prueba de MannWhitney; cuando se compararon las medias de más de dos grupos, se utilizó la prueba de Kruskal-Wallis; y en el caso de las correlaciones, se utilizó el coeficiente de correlación de Spearman.

El análisis de correlación con cada una de las variables sociodemográficas significativas del estudio, edad $(\mathrm{P}=0,078)$, sexo $(\mathrm{p}=0,484)$, estado civil $(\mathrm{p}=0,105)$, nivel educacional $(\mathrm{p}=0,538)$, número de años trabajados $(\mathrm{p}=0,077)$, percepción del estado de salud $(\mathrm{p}=0,485)$, presencia de enfermedades $(\mathrm{p}=0,116)$ y discapacidad física $(\mathrm{p}=0,248)$, arrojó que no existe correlación de estas variables con el nivel de dificultad para la decisión de jubilar, dado que no mostraron significancia estadística.

No obstante lo anterior, se pudo observar para la variable edad y sexo que la muestra está compuesta preferentemente por hombres, 
y que la edad en que jubilaron los encuestados se ha prolongado en promedio 2,88 años más, respecto de la edad legal de pensión, que es 65 para hombres y 60 para mujeres; en concreto los hombres trabajaron en promedio 2,10 años más, y las mujeres 4,14 años más. Este hallazgo concuerda con datos de la Organización de Cooperación y Desarrollo Económico, que plantea que la edad de retiro en Chile en las mujeres alcanza los 70,4 años.

Tabla N² Dificultad para la decisión de jubilar por Sexo

\begin{tabular}{|l|l|l|l|}
\hline Sexo & N & & Sx \\
\hline Femenino & 13 & 23,38 & 8,09 \\
\hline Masculino & 21 & 24,62 & 10,04 \\
\hline Total & $\mathbf{2 4 , 1 5}$ & $\mathbf{3 4}$ & $\mathbf{8 , 7 5 6}$ \\
\hline
\end{tabular}

Fuente: Tesis de Magíster "La decisión de jubilar, dificultades y factores asociados en Personal Universitario de Concepción”. Marzo 2016.

Al relacionar la variable sexo, según tabla $\mathrm{N}^{\circ} 2$ se observó en la muestra tendencia a que las mujeres tienen menor dificultad para la decisión de jubilar, una explicación podría ser que a pesar de la incorporación de la mujer al mercado laboral, la mayoría de los hombres continúa con la responsabilidad de ser el principal sustento del hogar, y siente que una vez jubilado debe continuar en una actividad laboral remunerada.

En relación a la variable "conocimiento sobre el sistema previsional", se midió por una escala con un rango desde los 08 hasta los 24 puntos (quienes presentan mayor conocimiento). $\mathrm{El} \mathrm{N}$ fue 30 , dado que no se consideró a los 04 afiliados al IPS, por tratarse de preguntas referidas al sistema de AFP. La escala muestra los siguientes resultados:

Tabla 3. Dificultad para la Decisión de Jubilar por Conocimiento sobre el Sistema Previsional

\begin{tabular}{|l|c|c|c|c|c|c|c|}
\hline & N & Media & Mediana & Moda & Desv. Tip. & Mínimo & Máximo \\
\hline $\begin{array}{l}\text { Jubilados } \\
\text { de AFP }\end{array}$ & 30 & 14,37 & 14,00 & 9 & 3,728 & 08 & 22 \\
\hline
\end{tabular}

Fuente: Tesis de Magíster "La decisión de jubilar, dificultades y factores asociados en Personal Universitario de Concepción” 2016. 
Se observó que las personas con distinto conocimiento previsional no presentan diferencias estadísticamente significativas $(\mathrm{p}=$ 0.662) con la dificultad para tomar la decisión de jubilar. A su vez, se pudo observar que la población en estudio mostró una media de 14,37, ubicándose bajo esta media el 53,3\% de las personas, y sobre esta media un $46,7 \%$, lo que muestra tendencia al bajo conocimiento previsional.

Respecto a la variable "satisfacción con el sistema previsional" de los encuestados, se encontró que los resultados muestran que no existe diferencia estadísticamente significativa con la dificultad para la decisión de jubilar ( $\mathrm{p}=0.662)$, en tanto, en el análisis univariado se pudo observar que en una escala de 2 a 6 (rango desde mayor insatisfacción a mayor satisfacción) la media se ubica en 2,5, concentrando el $50 \%$ de las personas, y en posición bajo 4 se ubica el 76,5\% de los encuestados, lo que muestra una distribución de resultados claramente polarizados, entre los que están Muy Insatisfechos y los que están Muy Satisfechos con el sistema de pensiones al que pertenecen. Cabe destacar que en los dos últimos tramos de satisfacción se ubican 04 encuestados pertenecientes al antiguo sistema de pensiones IPS, según tabla $\mathrm{N}^{\circ} 4$.

Tabla Nº4 Dificultad para la Decisión de Jubilar por Satisfacción con el Sistema Previsional

\begin{tabular}{|l|c|c|c|c|}
\hline $\begin{array}{l}\text { Menor a mayor } \\
\text { satisfacción }\end{array}$ & N & $\%$ & & Sx \\
\hline Mayor Insatisfacción & 17 & 50 & 23,82 & 11,075 \\
\hline Satisfacción deficiente & 4 & 11,8 & 21,50 & 4,655 \\
\hline Indiferente & 5 & 14,7 & 24,60 & 7,197 \\
\hline Satisfacción suficiente & 7 & 20,6 & 26,00 & 6,325 \\
\hline Mayor satisfacción & 1 & 2,9 & 25,00 & - \\
\hline Total & $\mathbf{3 4}$ & $\mathbf{1 0 0}$ & $\mathbf{2 4 , 5 0}$ & $\mathbf{8 , 7 5 6}$ \\
\hline
\end{tabular}

Fuente: Tesis de Magíster "La decisión de jubilar, dificultades y factores asociados en Personal Universitario de Concepción”. Marzo 2016. 
La pregunta abierta: ¿cuáles fueron los factores más importantes considerados por Ud. para tomar la decisión de jubilar?, se planteó como objetivo complementar el estudio y conocer desde una perspectiva más personal, abierta y profunda los factores asociados a la dificultad para la decisión de jubilar, desde la experiencia subjetiva de las personas. Se logró construir conceptos que permitieron comprender las variables analizadas, y conocer los hallazgos relacionados con cada una de las siguientes variables, de acuerdo a tabla $\mathrm{N}^{\circ}$ :

Tabla $\mathrm{N}^{\circ} 5$ Categorías asociadas a variables del Estudio

\begin{tabular}{|l|l|c|}
\hline Variables & Categoría encontrada & $\%$ \\
\hline Edad & "cumplimiento de edad" & 14,2 \\
\hline$N^{\circ}$ de años trabajados & "años de trabajo suficientes" & 20,6 \\
\hline Salud & "problemas de salud”, "retirarse en buen & 20,6 \\
& estado de salud" & 17,6 \\
\hline Nivel de conocimiento & "proceso planificado" & 20,6 \\
\hline Satisfacción con el sistema & "asumir brusca reducción & 23,5 \\
previsional & del sueldo" por bajas pensiones & \\
\hline
\end{tabular}

Fuente: Tesis de Magíster "La decisión de jubilar, dificultades y factores asociados en Personal Universitario de Concepción”. 2016.

Respecto de la edad, los encuestados asociaron la jubilación como evento normativo al cumplir los 60 años y 65 años respectivamente, y coincide con el inicio de la etapa de vejez, que expone además al individuo a una verdadera encrucijada de crisis y tareas, caracterizada por la integridad versus desesperanza (Erickson 1997). En relación al $\mathrm{N}^{\circ}$ de años trabajados, un 20,6\% lo consideró suficiente, en función de que el adulto sano consume la mayor parte de su tiempo de vigilia y de sus energías dedicado a las actividades de producir y/o distribuir bienes y servicios.

Respecto a la salud, los encuestados en un 20,6\% manifestaron haberse retirado por problemas de salud que los tenían limitados en las actividades diarias normales; y en 17,6\% la salud fue asociada por los encuestados a incertidumbre frente al futuro declive de la salud y gasto creciente, y las dificultades que deben 
enfrentar ante las pérdidas de los beneficios de salud respecto de su condición de activos, y también a la amenaza de aparición de enfermedades.

El nivel de conocimiento fue homologado a la categoría "proceso planificado". En este sentido la etapa de transición a la jubilación se logra enfrentar de mejor forma si previamente la persona participa de un proceso de preparación integral para ello. Se considera que desarrollo y envejecimiento han de entenderse como procesos simultáneos y permanentes durante la vida, en los cuales se conjugan ganancias y pérdidas, así como múltiples influencias y orientaciones (Dulcey-Ruiz, 2002).

En relación a la satisfacción con el sistema previsional, un 23,5\% manifestó en forma expresa dificultades para asumir la pensión que obtendría como jubilación, por su bajo monto. En este sentido, los aspectos negativos asociados al proceso de la jubilación de los encuestados, estuvieron dados principalmente por lo material y económico: los encuestados expresan que una vez que "asumieron y se resignaron" respecto del monto de las pensiones que percibirían, tomaron la decisión de jubilar.

Las "nuevas categorías" identificadas en la pregunta abierta o los nuevos hallazgos indican como factores relevantes para la decisión de jubilar los siguientes:

- Los incentivos económicos asociados al retiro por jubilación (73,5\%), siendo éstos la Renta Vitalicia en la Universidad de Concepción, la Indemnización especial por años de servicio en la Universidad Católica de la Ssma. Concepción.

- La toma de control de su propio tiempo (35\%)

- El descanso y disfrutar de la vida (32,3\%)

- Dejar espacio a las nuevas generaciones $(25,7 \%)$

- Trabajar en forma libre (25,7\%)

- Asumir reducción de los ingresos por bajas pensiones (23,5\%)

- Problemas de salud (20,6\%)

- Años de trabajo suficiente (20,6\%)

- Cumplimiento de un ciclo o etapa de vida (17,6\%) 
- Reintegración en la familia (17,6\%)

- Retirarse en un buen estado de salud (17,6\%)

- Cumplimiento de la edad (14,2\% )

- Término de la educación de los hijos $(11,7 \%)$

- Acogerse a jornada parcial (11,7\%).

Se debe enfatizar la incidencia que ha tenido en la decisión de jubilar la categoría "incentivos económicos asociados al retiro laboral por jubilación”, factor presente en el 73,5\% de los encuestados.

La seguridad económica es esencial para una jubilación satisfactoria e independiente, en un contexto en que la pensión de un jubilado implica una reducción muy considerable de los ingresos económicos a percibir, y no les permite mantener el mismo estándar de vida que tenían cuando trabajaban. Esta variable se asocia con la variable satisfacción con el sistema de pensiones, que concentró el $76.5 \%$ en la mayor insatisfacción.

El sistema previsional está entregando pensiones con tasas de reemplazo que alcanzan el promedio de 51,8\% del último sueldo percibido (OCDE, 2015), en este sentido cobran gran relevancia para el futuro jubilado los incentivos económicos por término de etapa laboral.

A pesar de que los análisis de correlación entre las variables de estudio no resultaron estadísticamente significativas, se pudo realizar un análisis cualitativo de la pregunta abierta, y establecer otras categorías de variables, que en forma individual o en conjunto determinaron la decisión de jubilar en los encuestados.

\section{Conclusiones}

Si bien la muestra es pequeña, igualmente fue posible hacer análisis importantes frente al tema. Aunque no hubo significancia estadística de la variable dificultad para tomar la decisión de jubilar en relación a las variables demográficas, al nivel de conocimiento previsional y al nivel de satisfacción con el sistema previsional, la pregunta abierta permitió conocer mediante un análisis cualitati- 
vo los factores asociados a la decisión de jubilación sustentados en el relato de los propios encuestados.

En referencia a la dificultad para tomar la decisión de jubilar respecto a las variables demográficas, sexo y edad de jubilación de los encuestados, los resultados del estudio mostraron que se ha prolongado en promedio 2,88 años más, considerando 65 años los hombres y 60 años las mujeres, lo que confirma que las personas en Chile están retrasando su jubilación, y uno de los factores es la baja tasa de reemplazo (OCDE "Pensions at a Glance 2013"); por lo tanto, se considera que un bajo monto de pensión es un factor que a la persona le genera una mayor dificultad para tomar la decisión de jubilar.

Concordante con lo anterior, el estudio arrojó relación entre la dificultad para tomar la decisión de jubilar y la variable satisfacción con el sistema de pensiones, dado que mostró que el 76\% de los encuestados se concentró en el rango de mayor insatisfacción con el sistema de pensiones, posición confirmada por el 73,5\% de los encuestados, que manifestaron que el factor más importante que determinó su retiro, fue el "incentivo económico asociado al retiro laboral".

La Comisión Asesora Presidencial sobre el Sistema de Pensiones, presidida por David Bravo, hace referencia a que existe un importante descontento respecto de la calidad de las pensiones, ya que luego de años de esfuerzo en el mercado laboral, los trabajadores ven cómo el nivel de la pensión que reciben, no se condice con sus expectativas, es decir, es muy bajo el monto de su pensión. Por otra parte, la expectativa de vida de hombres y mujeres, junto con la edad de jubilación, son elementos relevantes para los montos de pensión. En Chile, la expectativa de vida de las mujeres ha sido mayor que la de los hombres y continúa en aumento (Comisión Asesora Presidencial, 2015).

En el presente año entraron en vigencia nuevas tablas de mortalidad calculadas por la Superintendencia de Valores y Seguros y por la Superintendencia de Pensiones. Estas nuevas tablas de mortalidad permiten proyectar que las mujeres afiliadas al sistema de 
pensiones que hoy tienen 60 años (edad legal de jubilación) vivirán en promedio hasta los 90,31 años, esto es 1 año y 2 meses más respecto de las tablas vigentes al 2015. La mayor sobrevida, involucra una baja promedio de $2,2 \%$ en las pensiones, respecto de las pensiones calculadas con la tabla anterior (2015). No obstante, ese porcentaje de la pensión no se pierde, sino que es redistribuido para cubrir la mayor longevidad y evitar que los fondos se agoten antes del final del periodo estimado.

En el caso de los hombres que hoy tienen 65 años (edad legal de jubilación), se estima que su esperanza de vida llegará en promedio a 85,24 años, esto es 7 meses más respecto de la tabla anterior. El efecto sobre las pensiones se reflejará en una baja promedio de $2,1 \%$ que, al igual que en el caso de las mujeres, es distribuido durante más años de pensión para cubrir la mayor sobrevida (Superintendencia de Pensiones, 2016).

En virtud de los antecedentes anteriores y con el objeto de aumentar los fondos acumulados, en especial el de las mujeres, se plantea realizar ajustes a la edad legal de jubilación en función del aumento de las expectativas de vida de la población, considerando al mismo tiempo, políticas o estrategias de apoyo al trabajador mayor (Comisión Asesora Presidencial, 2015). La principal propuesta apunta a equiparar la edad de retiro entre hombres y mujeres en forma gradual (10 años), que incluye una revisión periódica de la edad de jubilación.

Al mismo tiempo estas medidas se consideran en su implementación complementadas con estrategias que buscan aumentar la participación laboral de las personas mayores y su calidad, así como también eliminar la existencia de desincentivos a trabajar en las personas mayores por el funcionamiento del Pilar Solidario. Al equiparar las edades de retiro, también se busca terminar con las diferencias de género de los actuales parámetros del sistema, permitiendo que hombres y mujeres tengan el derecho a permanecer en el mercado del trabajo por el mismo tiempo, y con iguales condiciones de retiro (Comisión Asesora Presidencial, 2015). 
Otra propuesta en este sentido plantea promover participación laboral de las personas mayores, más allá del aumento en la edad de jubilación, pero tomando en cuenta que un aumento podría tener efectos no deseados en la salud y bienestar psicológico de las personas mayores. De especial importancia es que la edad de jubilación no supere la edad en que distintos grupos de personas todavía son aptos para trabajar y que vaya acompañada de incentivos fuertes a los empleadores para contratar y retener a los trabajadores mayores. Entre ellos, subsidios a las contribuciones de los empleadores que sean proporcionales a los años adicionales de trabajo por sobre la edad actual de jubilación, así como regulación laboral que prohíba de forma más estricta la discriminación por edad (Calvo, 2016).

La variable conocimiento previsional mostró que la población en estudio se ubica en una tendencia bajo la media en un 53,3\%, pese al nivel educacional de los encuestados. La encuesta de "percepción y opinión del sistema de pensiones en Chile", arrojó entre sus resultados que el nivel de conocimiento de la ciudadanía sobre los principales componentes del sistema de pensiones y la Reforma previsional del año 2008 es más bien escaso, y es posible establecer que el actual sistema de pensiones es regresivo en tanto se adecua más a los encuestados con mayores niveles educacionales y de ingreso. Esta encuesta se realizó en el marco de la labor de la Comisión de Pensiones, se aplicó en todo el país a 3.696 ciudadanos, en 2014, y tuvo como objetivo identificar el nivel de conocimiento y evaluación de la ciudadanía, sobre los principales componentes del Sistema de Pensiones y la Reforma previsional del año 2008 (Informe Final Statcom, 2014).

En el contexto de la presente investigación, se observó que la fase previa a la jubilación se caracterizó por sentimientos de "incertidumbre", según señalaron los propios encuestados, en función del escaso conocimiento que poseían sobre los temas asociados al proceso de jubilación, principalmente el aspecto material y económico, que los expuso a sensación de temor e inseguridad y a una imperiosa necesidad por conocer los montos de pensión, con el 
fin de organizar las finanzas, orientados a disminuir el endeudamiento y fomentar el ahorro, en conjunto con la familia.

De lo anterior se concluye que para que la jubilación sea valorada como una oportunidad de desarrollo, exige la necesidad de Políticas Públicas orientadas a la preparación para la jubilación, para quienes estén cercanos al cese laboral normativo, así como también del empleo de diversas estrategias de intervención para quienes, ya jubilados, no logran una buena adaptación a su situación, como un recurso ineludible para una correcta adecuación y vivencia equilibrada durante la jubilación. "Se trata de que el sujeto asuma la jubilación como un derecho tras una vida de trabajo, y no como una imposición forzada que lo separa del normal desarrollo de su vida cotidiana” (Rodríguez, 2006: 28).

Este estudio es un aporte a las ciencias sociales, en un contexto caracterizado por la insatisfacción de las personas y la necesidad de prepararlo para una nueva etapa de vida, desde donde surgen demandas a las ciencias sociales, en concreto en las áreas de:

La medicina, por la necesidad de preparar médicos geriatras especialistas en enfermedades de los adultos mayores y ayudar a controlar los déficits de la salud propios de la edad.

La psicología, para que apoye en la contención de los miedos y eliminar conductas de negación, utilizadas para eludir enfrentamiento a las limitaciones propias de la edad. Ejercitar la mente para mejorar la memoria, las habilidades sociales que faciliten las interacciones con el nuevo medio en el que se tiene que vincular. Enseñar y entregar técnicas apropiadas según la constitución física, para que pueda desempeñar nuevas funciones.

La sociología, porque la configuración de la sociedad ha aumentado el número de personas mayores, lo que va a demandar una readecuación de las actividades comerciales para atender las necesidades de este segmento. Generar conductas positivas de enfrentamiento a la nueva realidad. Buscar un nuevo sentido de vida, entre otros. 
Desde el Trabajo Social se propone impulsar políticas públicas que generen instancias de preparación para la jubilación, destinada a los trabajadores mayores. Estas políticas deben tener como objetivo apuntar al diseño de estrategias de intervención directa y de planificar proyectos, que permitan la ejecución de programas para abordar la problemática que se le presenta al adulto mayor, en el momento de su jubilación por vejez, y también por causal de invalidez, ante el retiro temprano de la actividad laboral.

Como política de Estado, se estima que el Ministerio del Trabajo y Previsión Social debería ser el organismo para definir los principales lineamientos de una política de apoyo al retiro laboral, y en su ejecución se proponen instancias como el Instituto de Previsión Social, que en convenio de cooperación técnica y de capacitación con Cajas de Compensación, con instituciones gremiales públicas y/o privadas, y con los propios empleadores, brinde herramientas teórico-prácticas al personal de dichas instituciones, tanto para descentralizar y multiplicar los programas, como para desarrollar talleres de capacitación en forma directa con el trabajador en situación de retiro laboral. Lo anterior, con el fin de apoyarlos en un nuevo proyecto de vida personal y en relación con su entorno social, como asimismo contribuir al enfrentamiento positivo de los cambios que se generan en esta etapa de vida.

Las acciones en este sentido, deben considerar lineamientos en las políticas de Recursos Humanos de las organizaciones, tendientes a la revalorización del adulto mayor, de transferencias del conocimiento a los trabajadores más jóvenes, previo al despegue laboral; como asimismo, integrar y capacitar al personal de Recursos $\mathrm{Hu}-$ manos de las instituciones que trabajen con personas en procesos de pre-jubilación.

En el sistema de pensiones vigente cobra relevancia la información previsional desde los comienzos de la vida laboral y en forma regular, a fin de crear conciencia de la preparación para la vejez. Se considera que la información que entregan las administradoras de fondos de pensiones no siempre resulta cercana y comprensi- 
ble para el trabajador, por ende una instancia complementaria de apoyo a esta información podrían ser los empleadores, a través de las áreas de Recursos Humanos.

El trabajo social puede contribuir desde distintas áreas a mejorar el nivel de conocimiento previsional, y con ello al grado de satisfacción de las personas al jubilar. Se requiere por tanto que el trabajador social se ocupe del tema, con el fin de educar y orientar a las personas en las distintas etapas de su vida laboral, conducentes a que el trabajador logre un proceso de jubilación asociado a un envejecimiento activo.

Dado que la vejez es una etapa natural e inexorable por la que atraviesa todo aquel que no muere joven, es necesario mejorar el estereotipo acerca de esta etapa de vida. Por lo tanto, los agentes principales de este proceso, es decir, la familia, la escuela, los medios de comunicación de masas, etc., deberían intentar transformar a través de la persuasión y del ejemplo, las actitudes negativas en positivas. Este cambio redundará en una mejor calidad de vida para las personas mayores actuales y futuras y solo así los esfuerzos de la medicina para prolongar la vida, tendrán un sentido cabal (Rodríguez, 2006).

Sería interesante realizar una investigación con las personas de las mismas instituciones en que se realizó el estudio, que no se han acogido a jubilación por edad legal, y que se encuentren en etapa de prejubilación.

Este es un tema propio de los Trabajadores Sociales, por el momento actual y dada la contingencia ante la opinión pública. Por lo tanto, debería ser considerado con la relevancia que amerita en la formación de pre y postgrado de los estudiantes de Trabajo Social.

Finalmente, se concluye con la siguiente cita: "No queremos un país en que la tercera edad sea sinónimo de vulnerabilidad o desprotección, y donde la jubilación, en lugar de descanso, traiga aflicciones a las personas...." (Discurso de Michelle Bachelet, 29 de abril 2014). 


\section{Bibliografía}

Asociación de AFP Chile, 2016. Recuperado el 08 de agosto 2016 de http://www.aafp.cl/el-sistema/el-sistema-afp/caracteristicas-del-sistema/

Bárcena, A. (2011). Comisión Económica para América Latina y el Caribe (Cepal). America Latina Envejece. Recuperado el 8 de agosto 2016. de http://www.americaeconomia.compolitica-sociedad/sociedad/america-latina-envejece

Begoña, V. (2007). Teoría de la Decisión: Decisión con Incertidumbre, Decisión Multicriterio y Teoría de Juegos. Universidad Complutense de Madrid.

Bruguera, M. y Calvo, A. (2012). Preparándonos para la jubilación. Una responsabilidad individual y de los colegios profesionales. Medicina clínica, 139, pp. 38-41.

Calvo, E., Sarkisian, N. y Tamborini, Ch. (2013). "Causal Effects of Retirement Timing on Subjective Physical and Emotional Health." Journal of Gerontology: Social Sciences 68(1):7384. Recuperado de http://dx.doi.org/10.1093/geronb/ gbs097

Calvo, E. (2016). Reforma al sistema de pensiones Chileno: Consideraciones No Pecuniarias, Documento de trabajo $N^{\circ} 27$, Instituto de Políticas Públicas, Universidad Diego Portales.

Casen (2013). Encuesta de Caracterización Socioeconómica Nacional. Recuperado el 8 de agosto de 2016 de http://observatorio. ministeriodesarrollosocial.gob.cl/documentos/Presentacion_Resultados_Encuesta_Casen_2013.pdf

Centro Latinoamericano y Caribe de Demografía (CELADE) (2009). Envejecimiento, derechos humanos y políticas públicas, División de Población de la CEPAL.

Comisión Asesora Presidencial (2015). Informe Final sobre el sistema de Pensiones. Recuperado el 8 de agosto de: http://www. comision-pensiones.cl/Documentos/GetInforme

Diario electrónico El Mostrador: Consejero del banco central lanza alerta al sistema de AFP. Transición demográfica lo hace una bomba de tiempo. Recuperado de http://www.elmostradormercados.cl/destacados/consejero-del-banco-centrallanza-alerta-al-sistema-de-afp-transicion-demograficalo-hace-una-bomba de tiempo, el 16 de octubre de 2016.

Diario electrónico El Mostrador: La impactante y triste realidad de las pensiones en Chile. Opinión. http://www.elmostradormercados.cl/destacados/opinión-la-impactante y triste realidad de las pensiones en chile. Recuperado el 8 de enero 2014. 
Diccionario de la Lengua Española-Real Academia Española (2014). Recuperado el 15 de junio 2014, de http://www.rae.es

Dulcey-Ruiz, Uribe, C. (2002). Psicología del ciclo Vital: hacia una visión comprehensiva de la vida humana en Revista Latinoamericana de Psicología. Vol. 34-Nº 1-2, p. 17-27.

Erickson, E. (1997). El Ciclo Vital Completado. España, Ediciones Paidós Ibérica, S.A.

García-Rodríguez, Sarabia-Cobo, C., Fusari, A., Villarino, A., Ellgrig, H., Molina, J. (2010). Envejecimiento activo, Capacidades cognitivas y Bienestar personal. Universidad Nacional de Educación a Distancia. Madrid, Viguera Editores.

Guillemard, A. (2011). Las nuevas orientaciones de la protección e inversión social en Europa. Ponencia III Congreso Personas mayores, ciudadanía y empoderamiento. De la investigación a la acción. Ciedess y Pontificia Universidad Católica de Chile.

Martens, J. (2014). HelpAge International. Índice global del envejecimiento. Recuperado el 08 de agosto de 2016 de: http://www.helpage.es/noticias/lanzamos-el-ndice-global-del-envejecimiento.

Izar, J.M., Izar, J., Abreu, M. (2010). Factores que influyen en la decisión de jubilación del personal de la Universidad Autónoma de San Luis Potosí, Revista de Investigación, Núm. 10, pp. 1-22.

Leeson, G. (2011). Discurso en visita a Universidad Autónoma de México. (UNAM). Inglaterra: Universidad de Oxford.

Rincón, S. (2011). "El Proceso de Jubilación del Docente: Una Mirada Desde la Orientación Psicosocial". La Universidad Del Zulia, Maestría en orientación. Línea de investigación Teoría y Praxis de la Investigación Cualitativa. www.rincon58.soraya@gmail.

Ochoa, C. (2015). Concepto bola de nieve. Recuperado el 22 de mayo de 2016. http://www.netquest.com/blog/es/muestreo-bolanieve/

OPS-OMS, 2015. Envejecimiento activo y saludable, Visión OMS/OPS Encuentro México Japón, febrero 2015. Recuperado el 8 de agosto de 2015 de: http://www.geriatria.salud.gob. $\mathrm{mx} /$ descargas/mexico japon/03_AYALA_envejecimiento_activo.pdf

Organización Iberoamericana de Seguridad Social (2011). Perspectiva general: La situación de los adultos mayores en la Comunidad Iberoamericana. Documento de la Secretaría General.

Rodríguez, N. (2007). Actitudes hacia la Jubilación, Revista de Psicología $y$ creencias afines Interdisciplinaria, vol. $24, \mathrm{n}^{\circ} 1$, pp. 5-42. 
Sarabia, C., Delgado, A. y Castañedo, P. (2011). A vueltas con la Jubilación, Gerokomos, n ${ }^{\circ} 22$ (2), pp. 72-74, recuperado de http://scielo.isciii.es/pdf/geroko/v22n2/comunicacion_ breve.pdf, el 18 de octubre de 2016.

Simon, H. A. (1957). Administrative Behavior: A case of Decision Making Process in Administrative Organization, second edition, NY, NY McMillan.

Statcom (2014). Informe Final Encuesta de opinión y percepción del Sistema de Pensiones en Chile. Preparado para: Comisión Asesora Presidencial sobre el Sistema de Pensiones.

Stuardo, N., Zavala, M. y Merino, J.M. (2016). La decisión de jubilar, dificultades y factores asociados en Personal Universitario de Concepción. (Tesis inédita de magíster). Universidad de Concepción, Chile.

Superintendencia de Pensiones Chile (2015). Portal inicio, Orientación. Recuperado el 15 de enero de 2014, de: https://www.spensiones.cl/portal/orientacion/580/w3-propertyvalue-6069. html

Superintendencia de Pensiones Chile (2010). El Sistema de Pensiones Chileno, séptima edición, recuperado el 8 de agosto de 2016 de: https://www.spensiones.cl/portal/informes/581/ articles-7206_libroVIIedicion.pdf

Urrutia, M., Campos, S. y O'Connor, A. (2008). “Validación de una versión en Español de la Escala de Conflicto Decisional”, en Revista Médica de Chile vol.136, n 11, pags. 1439-1447.

Vial, J. (2013). Consejero del Banco Central. Recuperado el 15 de enero de 2013, de www.fundación chile21.cl

Villar, F. (2001). Psicología Evolutiva y Psicología de la Educación. Proyecto docente, Universidad de Barcelona, Madrid.

Villar, F. (2008). El enfoque del ciclo vital: hacia un abordaje evolutivo del envejecimiento. Asociación Multidisciplinar de Gerontología. Rev. Gerontología, actualización, innovación y propuestas, pp. 147-184.

Herbert, S. (2008). Proceso de toma de decisiones. Recuperado de http:// www.apuntesfacultad.com/proceso-de-toma-de-decisiones-herbert-simon.html el 16 de octubre de 2016. 